\title{
Analysis of Texture of Image with Statistics Method
}

\author{
Jishi Guan ${ }^{1,2, a}$, Xian Li, ${ }^{3, b}$, Yuguang Zhou ${ }^{3, c}$, Hongwei Shi ${ }^{4, d}$ \\ ${ }^{1}$ College of Communication Engineering, Jilin University, ChangChun,130025, China \\ 2 Testing and Simulation Dept, China Nuclear Power Technology Research Institute Beijing Division, \\ Beijing, 100085, China \\ ${ }^{3}$ State Grid Jilin Electric Power Co.Ltd,Changchun, 130001, China \\ ${ }^{4}$ Changchun University of Technology, Changchun, 130025, China \\ aemail: 12923605@qq.com, bemail: 3364546@qq.com, ${ }^{\mathrm{C}}$ email: \\ yuguang_zhou@163.com, ${ }^{\mathrm{e} e m a i l: q i t i a n d @ q q . c o m}$
}

Keywords: Image; Texture; Cross-correlate function; Distribute function of Gradient.

\begin{abstract}
This paper use two statistics methods to analyze the texture character in image. First it proposes cross-correlation function method. Most existing texture analyze method can only analyze the texture character of the whole image or a region in the image. But cross-correlation function can indicate the texture feature of single point. Further more the changing of light in same texture does influence the function. Second it proposes that Angle Distribute Function of Gradient can be used to analyze the direction of texture in an image or region. It can show a continuous angle feature of a single texture while traditional method can only provide discrete angle feature of texture.
\end{abstract}

\section{Introduction}

The concept of texture was concerned for a long time in image processing. It is an important feature in image, but it is difficult to describe. Further more the impress of texture for different individual is various, so it is difficult to give a definition for texture[1,2]. The common comprehension for texture is: any distribute or feature of object constitute, especially those involve character of appearance and sense of touching. In image processing[3,4], common comprehension for texture is: the feature that can indicate the regular pattern of point gray distribute in space.

Common texture analysis methods can be classified to three categories: method based on statistics, method based on contracture and method based on frequency spectrum. Some methods based on image model are proposed recently[5-9].

Existing texture analysis methods mostly use co-distribution to describe texture[10]. Co-distribution carries co-changing, but co-changing doesn't indicate co-distribution. Most existing texture analysis methods analyze texture feature by statistics the distribution information of an image or a region, and give the conclusion with the whole texture feature. They cannot measure texture information of a single point. Aimed at this problem, this paper proposed a method to measure texture feature of a single point. The method use the cross-correlation function of the data take from a line of which the point is the center point and other lines which coherent and parallel to this line. When the data is zeros-average and in same strength, the cross-correlation function will give a peak in the zero-point while it is plat in zero point if the point is not in a obvious texture.

According to the agreed definition for texture, texture can be measured by gray level distribute, further more, it can be measured by changing pattern of gray level. So the gradient information of an image can be used to analyze texture feature. Distribute function of gradient can provide a lot information of the whole image or a specified region. For a single texture, the angle distribution function of gradient can give the direction character of the texture in an image.

\section{Use cross-correlation function to analyze texture feature of single point}

If a point is in a texture, then the data from parallel lines must contains a same trend, when the 
data is transformed to zeros-average, then the cross-correlation function will give a peak at zero point. Before calculate the function, the data need to be transformed to zeros-average and value adjusted to same scope. For a data array $\mathrm{X}$ from a line, it can be transformed by formula 1 :

$$
X^{\prime}=\frac{X}{\bar{X}}-1
$$

Where $X$ is data array from a line, $\bar{X}$ stands for the average of the array, and $X^{\prime}$ is the transformation result. After the transformation $X^{\prime}$ is zero-average and average of absolute value of $X^{\prime}$ is 0.5 .

Definition for cross-correlation function(CCFT).To measure texture feature of a single point, first extract a data array from a line of which the aim point is the center point. Then extract some data array from a coherent and parallel line to this line. Data extracting can be indicate by fig 1

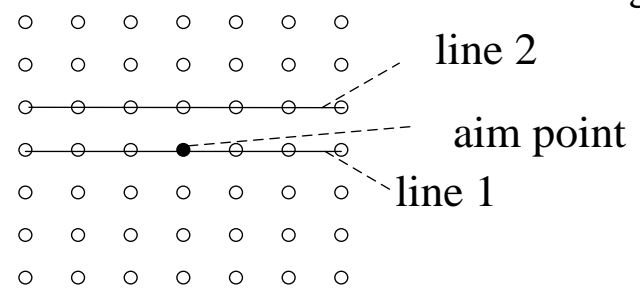

Fig 1 sample for data extracting

From line 1 and line 2, initial data array $X_{1}$ and $X_{2}$ can be extracted. Deal them with formu 1, get zeros-averaged data $X_{1}{ }^{\prime}$ and $X_{2}{ }^{\prime}$. Cross-correlation function of $X_{1}{ }^{\prime}$ and $X_{2}{ }^{\prime}$ can be used to analyze the texture character of the aim point.

So the cross-correlation function for texture character (CCFT) is defined as:

$$
\begin{aligned}
& X_{1}(k)=U(i+k, j) \quad X_{2}(k)=U(i+k, j+1) \quad k=-L \ldots L \\
& X_{1}{ }^{\prime}=\frac{X_{1}}{X_{1}}-1 \quad X_{2}{ }^{\prime}=\frac{X_{2}}{X_{2}}-1 \\
& T_{v}(i, j)=\sum_{0}^{2 L+1} X_{1}{ }^{\prime}(k) X_{2}{ }^{\prime}(k)
\end{aligned}
$$

Where $L$ stand for data length for texture analysis, at the same time, it stand for the strength for texture analysis. With bigger $L$, texture in wider region can be detected, but texture with small dimension would be ignored because the character would be covered.

The direction of a texture should refer to the direction that the data changes in a texture. So formu 2 can be used to analyze vertical texture. If a vertical texture placed at the aim point, then data change with a same pattern in $X_{1}$ and $X_{2}$, then CCFT can give a bigger value, otherwise the value of CCFT will be small.

Follow the definition of vertical CCFT, we can give horizontal CCFT:

$$
\begin{aligned}
& X_{1}(k)=U(i, j+k) \quad X_{2}(k)=U(i+1, j+k) \quad k=-L \ldots L \\
& X_{1}{ }^{\prime}=\frac{X_{1}}{X_{1}}-1 \quad X_{2}{ }^{\prime}=\frac{X_{2}}{\frac{X_{2}}{X_{1}}-1} \\
& T_{h}(i, j)=\sum_{0}^{2 L+1} X_{1}{ }^{\prime}(k) X_{2}{ }^{\prime}(k)
\end{aligned}
$$

Follow the upper definition, we can give 450 and 1350 CCFT : 


$$
\begin{aligned}
& X_{1}(k)=U(i+k, j+k) \quad X_{2}(k)=U(i+k+1, j+k+1) \quad k=-L \ldots L \\
& X_{1}{ }^{\prime}=\frac{X_{1}}{X_{1}}-1 \quad X_{2}{ }^{\prime}=\frac{X_{2}}{X_{2}}-1 \\
& T_{h v}(i, j)=\sum_{0}^{2 L+1} X_{1}{ }^{\prime}(k) X_{2}{ }^{\prime}(k) \\
& X_{1}(k)=U(i+k, j-k) \quad X_{2}(k)=U(i+k+1, j-k-1) \quad k=-L \ldots L \\
& X_{1}{ }^{\prime}=\frac{X_{1}}{X_{1}}-1 \quad X_{2}{ }^{\prime}=\frac{X_{2}}{\frac{X_{2}}{2}}-1 \\
& T_{v h}(i, j)=\sum_{0}^{2 L+1} X_{1}{ }^{\prime}(k) X_{2}{ }^{\prime}(k)
\end{aligned}
$$

Use formu 1-4 we can get $T_{h}, T_{v}, T_{h v}$ and $T_{v h}$ they can be used to analyze the texture character of the aim point.

Experiments for CCFT.Use the algorithm proposed in 1.1, we can test a image. For a given image, we calculate the CCFTs for every point. Then can draw the data pattern of the CCFTs. With a appropriate thresh we can segment the to 2-value and then give the segment result. Follow experiments are based on the images in Fig. 1:

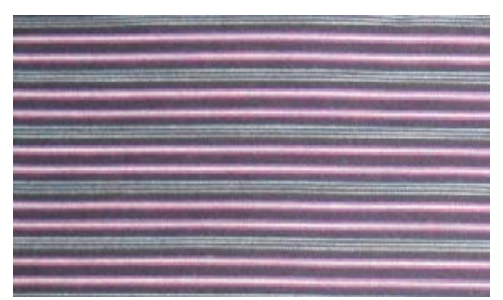

(a)

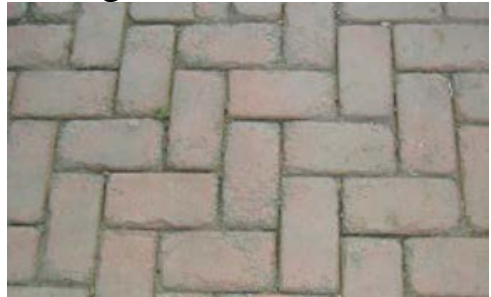

(b)

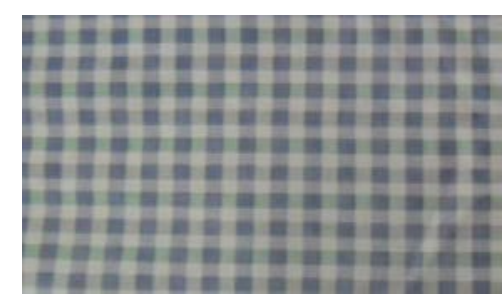

(c)

Fig. 1 Test resource

Experiment1: Basic experiment for CCFT, calculate CCFTs of the fig 1(a) and fig 1(c), and draw the data distribution with a three dimension mesh. The result is shown follow:
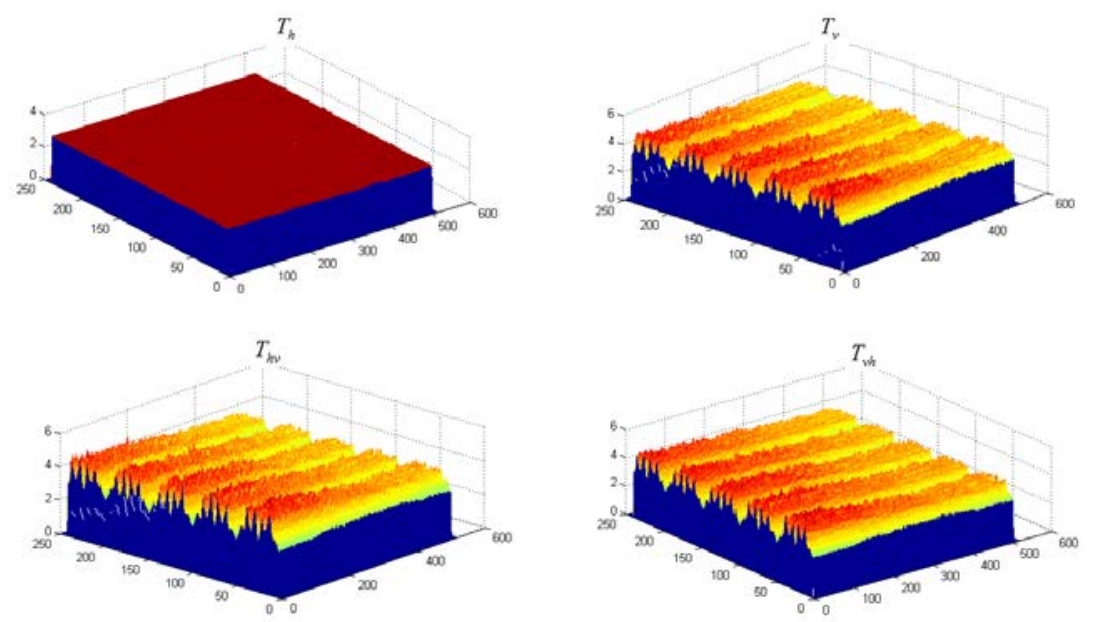

Fig 2 CCFTs of fig 1(a)

There is a obvious texture in fig 1(a). and then we can see that data in $T_{h}$ is plat and small, while data in $T_{h}$ is Dramatic changing. Because the direction of $45^{\circ}$ and $135^{\circ}$ is not orthogonal to the texture, so there is data changing in $T_{h v}$ and $T_{v h}$. 

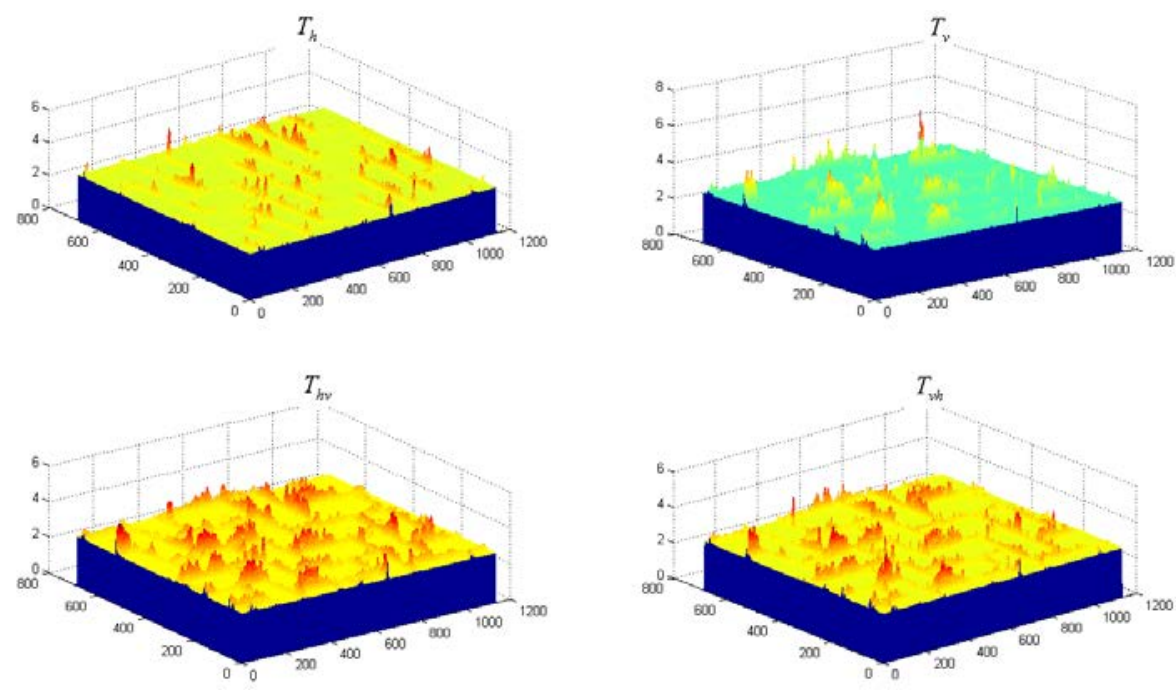

Fig. 3 CCFTs of fig 1(b)

There is vertical and horizontal texture at different region of the image, and in the $T_{h}$, the position with horizontal texture is obviously higher than other positions. And in $T_{v}$ the position with vertical texture is obviously higher than other positions. In $T_{h v}$ and $T_{v h}$, position with texture is higher than other positions.

Experiment 2: segment experiment.

We segment the CCFT result with Ostu thresh, we can get the points that with obvious texture character. The result is show in fig 4 and fig 5

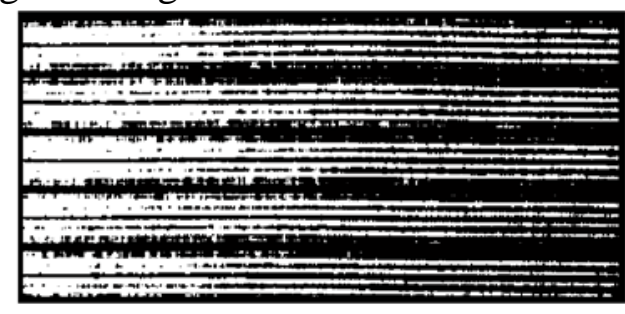

Fig . 4 Segment result of $T_{h}$ of fig 1(a)

From the segment result, the position with obvious texture is labeled with white color and others is labeled with black color. We can see regions with plat color changing is labeled black and regions with obviously texture is label white.

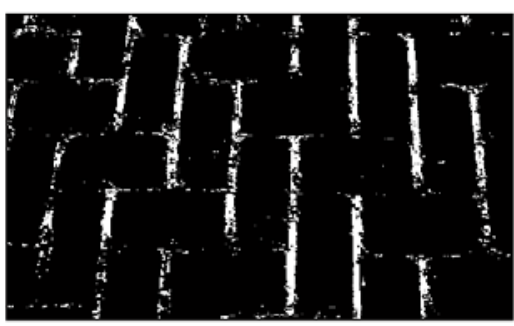

(a) $T_{h}$

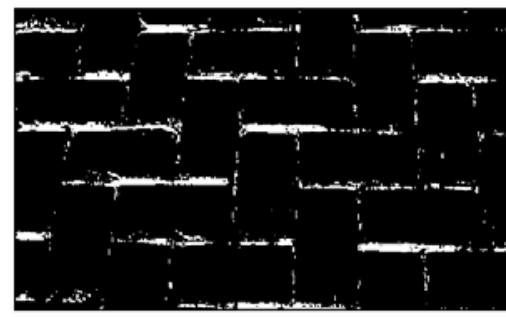

(b) $T_{v}$

Fig. 5 Segment result of CCFTs of fig 1(b)

In the result of $T_{h}$, regions with horizontal texture are labeled white and in result of $T_{v}$ regions with vertical texture are labeled white. In segment result of $T_{h}$, regions with vertical texture is slightly labeled and in segment result of $T_{v}$, regions with horizontal texture is slightly labeled.

From the experiments upper, CCFT is effective in texture analysis. It can indicate the texture 
character of a single point.

\section{Analyze texture character with GADF}

Distribution function of gradient is the histogram of gradient. It can be used to analyze the statistics feature. The gradient angle distribution function (GADF) is the histogram of gradient angle of an image. It can indicate the changing direction of an image. If there is some obvious texture with single direction, then there would be some peak value in GADF. Then texture with single direction can be indicate with GADF.

GADF is defined as follow[11]:

$$
D_{\theta}(\alpha)=\left.\operatorname{Count}(u)\right|_{\theta(i, j)>\alpha, \theta(i, j)<\alpha+\square \alpha}
$$

Where $\theta(i, j)$ stand for the gradient direction of point $(i, j)$. For texture analysis the contrast gradient direction maybe coming from the same texture. So the definition of $\theta(i, j)$ should be modified as:

$$
\theta(i, j)=\operatorname{angle}(g)=\arctan \left(\frac{g_{h}}{g_{v}}\right)
$$

Where $g_{h}$ and $g_{v}$ stand for horizontal and vertical gradient.

Texture character can be described as same distribution and same changing trend. A lot of textures bring out same changing direction. If there is a texture with single direction in a region, then the GADF of this region will show obviously peak value.

Experiment: use GADF to analyze the texture direction for fig 1(a),(b) and (d).

Image and the GADF is shown in Fig. 6:

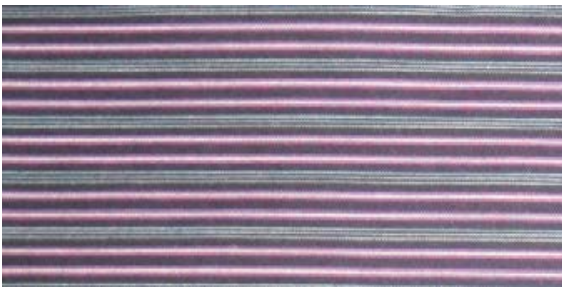

a) fig 1(a)

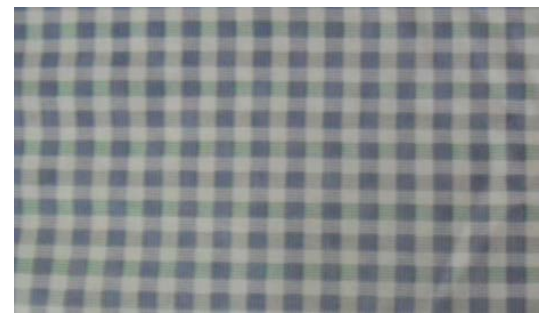

(c) fig 1(c)

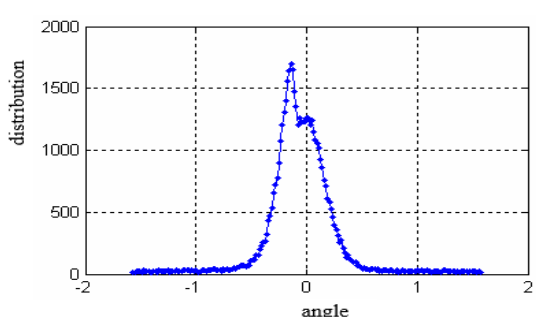

b)GADF of fig 1(a)

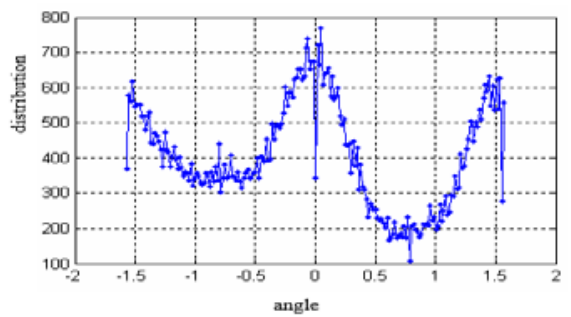

(d) GADF of fig 1(c)

Fig 6 GADF of resources

From the GADF, image fig 1(a) has a vertical texture, and the GADF has a peak value at 0 . Fig 1(b) have a texture at direction of $\frac{\pi}{4}$ and $\frac{-\pi}{4}$, and the GADF has two peak value at $\frac{\pi}{4}$ and $\frac{-\pi}{4}$.

Fig 1(d) has a texture at 0 and $\pi$, the GADF has peak value at $0, \pi$ and $-\pi$.

From these images, we can see that GADF can indicate the texture direction of images. Unlike traditional method, GADF can indicate continuous direction while most traditional method can only provide discrete angle of direction. 


\section{Conclusion}

This paper proposes two method to analyze texture character of image: method based on cross-correlation function (CCFT) and method based on gradient angle distribution function (GADF). Method based on cross-correlation function can give a measurement of the texture character of a single point in image. Method based on GADF can indicate the direction of texture in a image or region. Experiments verify the methods' efficiency in texture analysis.

\section{Reference}

[1] Zoltan Szantoi, Francisco Escobedo, etc. Analyzing fine-scale wetland composition using high resolution imagery and texture features[J]. International Journal of Applied Earth Observation and Geoinformation, Vol 23, 2013,204-212

[2] Topi Mäenpää, Matti Pietikäinen . Classi cation with color and texture: jointly or separately?[J] Pattern Recognition. Vol 37,2004,1629-1640

[3] Christoph Palm. Color texture classification by integrative Co-occurrence matrices [J]. Pattern Recognition, Vol 37. 2004,965-976.

[4] ZHANGShui-li,ZHENGXiu-ping,,LEIWen-li. Method of Color Image Retrieval Based on Quantified Color Space[J] vol 27(10),2010,194-196

[5] Rodrigo Pereira Ramos, Marcelo Zanchetta do Nascimento , Danilo Cesar Pereira. Texture extraction: An evaluation of ridgelet, wavelet and co-occurrence based methods applied to mammograms[J]. Expert Systems with Applications. Vol 39,2012,11036-11047.

[6] Charles Chubb. Texture-Based Methods for Analyzing Elementary Visual Substances[J]. Journal of Mathematical Psychology. Vol 43, 1999, 539-567.

[7] Hu Yong. Texture analysis and its applications for outdoor scene image[M]. Nanjing University of Science \& Technology. 2010.

[8] Hanbury, Mareotegui,Morphological segmentation on learned boundaries 〔 J] Image and Vision Computing,27(4), 2009,480-488,

[9] J Park, L Kurz, Unsupervised segmentation of textured images [J]. Information Sciences 92(1),1996,255-276.

[10] WAN G Yao Nan, WANG Shao Yuan, MAO Jian Xu. Image Texture Analysis Based on Fractal Dimension[J]. Journal of Hunan University(Natural Sciences), Vol 33(5),2006, 67-72.

[11] Shi Hongwei, The Theory of Microscopy Image Treatment and the Usage in the Detection for Animalcule in Food[M], Jilin University. 2012 